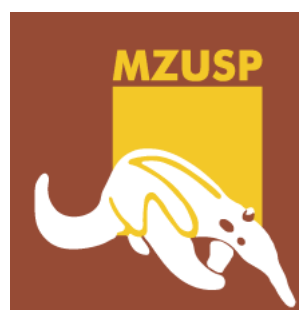

Museu de Zoologia da Universidade de São Paulo

Lívia Pires do Prado

\title{
Revisão taxonômica de Octostruma Forel, 1912 (Formicidae, Myrmicinae)
}

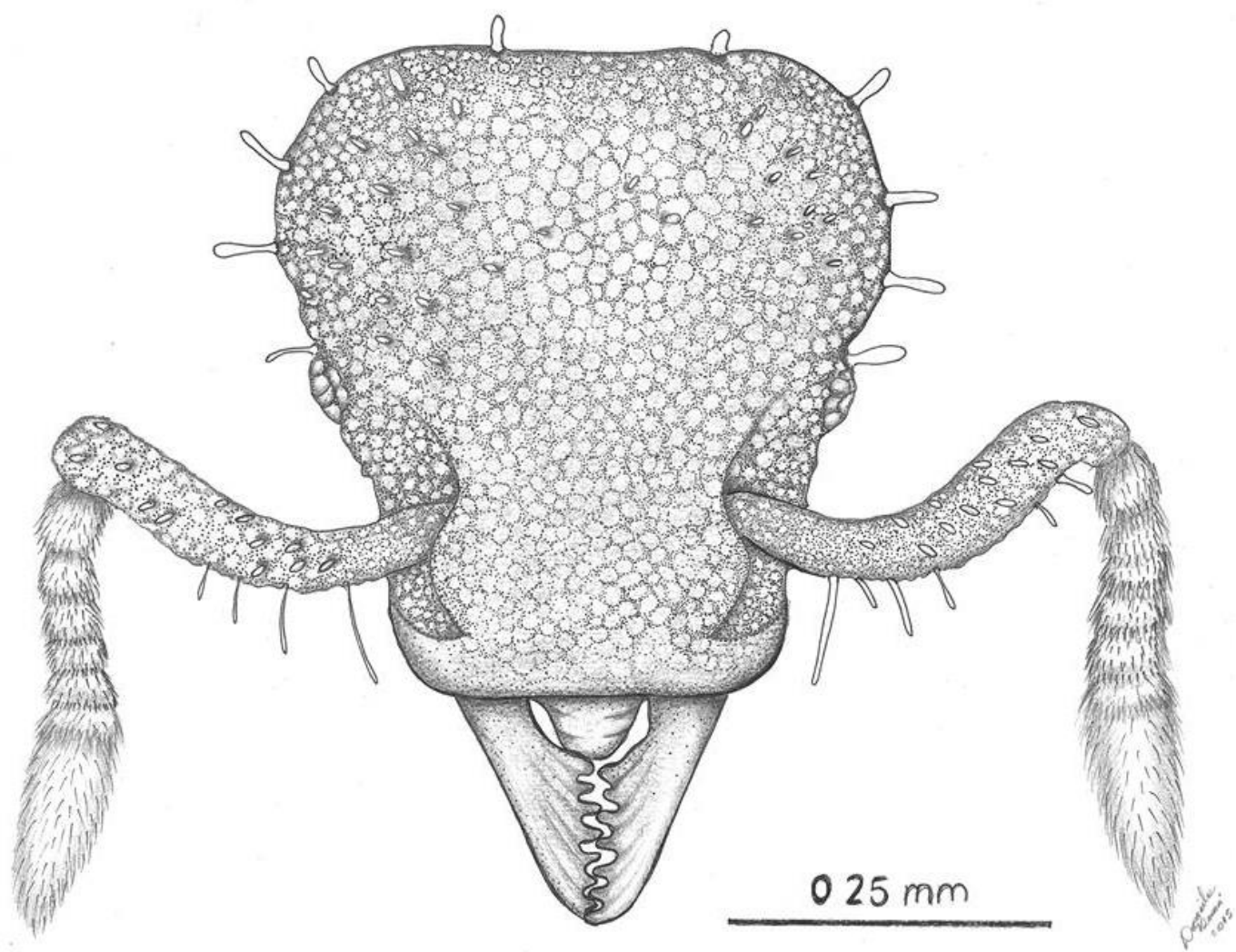

São Paulo

2015 


\section{Lívia Pires do Prado}

\section{Revisão taxonômica de Octostruma Forel, 1912 (Formicidae, Myrmicinae)}

Dissertação apresentada ao Programa de Pós Graduação em Sistemática, Taxonomia Animal e Biodiversidade do Museu de Zoologia da Universidade de São Paulo para a obtenção do título de Mestre.

Orientador: Prof. Dr. Carlos Roberto Ferreira Brandão.

São Paulo

2015 
Ficha Catalográfica

Prado, Lívia Pires do

Revisão taxonômica de Octostruma Forel, 1912

(Formicidae, Myrmicinae) / Lívia Pires do Prado; orientador Carlos Roberto Ferreira Brandão. - São Paulo, SP: 2015.

$222 \mathrm{fl}$

Dissertação de Mestrado - Programa de Pós Graduação em Sistemática, Taxonomia Animal e Biodiversidade do Museu de Zoologia da Universidade de São Paulo.

1.Octostruma Forel, 1916. 2. Formicidae, Myrmicinae. I. Brandão, Carlos Roberto Ferreira, orient. II. Título. 


\section{Banca Examinadora}

Prof. Dr. Carlos Roberto Ferreira Brandão (Orientador)

Julgamento Assinatura

Prof. Dr. Instituição

Julgamento Assinatura

Prof. Dr. Instituição

Julgamento Assinatura

Prof. Dr. Instituição 


\section{AGRADECIMENTOS}

Ao Prof. Dr. Carlos Roberto Ferreira Brandão, pela orientação e oportunidade, por fornecer toda infraestrutura necessária e um ambiente tão agradável para o desenvolvimento deste trabalho.

À Coordenação de Aperfeiçoamento de Pessoal de Nível Superior (CAPES) pela bolsa concedida para a realização desta dissertação.

Aos curadores e alunos das coleções mencionadas neste trabalho pela paciência e pelo empréstimo de material. Agradeço em especial ao Diego dos Anjos (UFOP) pelo envio do material de seu trabalho e ao Júlio Chaul (UFV) pelo envio de material de suas coletas e por sempre ser tão solícito em ajudar no que fosse preciso.

Aos Drs Bodo Dietz e John Longino por suas valiosas contribuições no estudo do gênero e ao Dr. Brian Fisher e toda equipe do AntWeb pela disponibilização das imagens das espécies de Octostruma.

Aos meus queridos amigos Rodolfo Probst e Camila Pereira... Por tudo que passamos juntos!

À minha querida amiga e parceira Mônica Ulysséa, pelos conselhos, ensinamentos no campo e pelas valiosas discussões em todas as etapas deste trabalho.

Às Dras Helena Onody e Kelli Ramos, pelo carinho e conselhos que foram sempre fundamentais em todos os momentos da minha formação.'

À minha amiga Helen Ferreira pelas importantes dicas sobre processamento de material e ao meu amigo Alvaro Santos pela amizade e pelas dicas nas edições das imagens.

Aos meus queridos amigos: Ricardo Eduardo Vicente pela fantástica parceria, conversas e incentivo nos momentos mais críticos; Alexandre Ferreira pelo carinho e consideração de sempre; Antônio Queiroz pelas conversas e piadas que certamente tornaram os dias mais leves; Roberto Padilha pelo incentivo e pela troca de ideias e à Fernanda Araújo que mesmo tão longe se faz tão presente em todos os momentos.

À minha talentosa amiga Daniela Bená por ter me presenteado com o lindo desenho da capa deste trabalho.

Ao maior coletor de Octostruma que este mundo já conheceu: Dr. Rogério Rosa da Silva! 
Ao Dr. Ricardo Kawada pelas conversas sobre morfologia e pelo incentivo no uso de outras técnicas.

Ao Fabiano Albertoni pelas lindas imagens de Octostruma em campo presentes neste trabalho.

Aos funcionários do MZSP, em especial ao pessoal da portaria por sempre me receber tão bem; à Dione Siripierre, pelas histórias, carinho e dedicação em ajudar no que fosse preciso; à Lara Guimarães pelo auxílio na obtenção das imagens de microscopia eletrônica e à Marta Maria, Omair Tizzot e Sonia Favaro por resolverem todos os problemas relacionados à pós-graduação.

Aos diversos amigos, de profissão ou não, que fizeram com que cada dia fosse melhor e mais divertido: Alex Quadros, Augusta Ribeiro, Bruna Barboza, Carolina Cuezzo, Débora Oliveira, Emília Albuquerque, Felipe Bueno, Gabriela Camacho, Hingrid Yara, Hosana Zotelli, Jader Oliveira, Joice Constantino, Lucimara Varejano, Luísa Santiago, Lyncoln Ferreira, Olívia Evangelista, Thalles Pereira, Thiago Silva, Tiago Carrijo, Victor Alarcon e Vinícius Ferreira.

E agradeço principalmente aos meus pais, Lailton e Maria Clara, por toda dedicação e por abrirem mão de muita coisa para que eu pudesse completar mais esta etapa e aos meus irmãos e exemplos, Guilherme e Paula por estarem sempre comigo e me ajudarem de tantas formas em todos os momentos que precisei. 
Aos meus pais e meus irmãos, com amor. 
"O bom cientista se revela e se realiza em seu laboratório, isso é lógico e ninguém escapa, mas o cientista feliz é aquele que se integra na mata virgem. A mata é uma dessas coisas em que o todo é mais do que a soma das partes. Não é só a luz, as plantas, os bichos, as vozes, mas é o todo que penetra na gente e que dá uma felicidade e uma tranquilidade imensa!"

Paulo Emílio Vanzolini - Calangos do Boiaçu, 1992. 


\section{RESUMO}

O gênero de formigas Octostruma Forel 1912 (Formicidae: Myrmicinae) está restrito à região Neotropical; suas espécies ocorrem do México até a Argentina, são predadoras de pequenos artrópodes e podem nidificar no solo, serapilheira e em epífitas. No presente trabalho, a taxonomia do gênero é revista com base no exame de cerca de 4.000 exemplares. Reconheço 43 espécies de Octostruma, 34 espécies previamente conhecidas são consideradas válidas e redescritas e, portanto, nove são reconhecidas como novas. Além disso, indivíduos de castas e sexos ainda não registrados (machos, gines e ergatóides) são aqui descritos pela primeira vez para diferentes espécies. Novos registros de localidades expandem a distribuição geográfica conhecida para parte das espécies e informações sobre a biologia das espécies a partir de dados de rótulo e observações de campo, somados às poucas informações encontradas na literatura são compiladas neste trabalho. Uma chave de identificação para as operárias de todas as espécies do gênero é fornecida.

Palavras-chave: Formigas, Myrmicinae, Octostruma, revisão taxonômica, região Neotropical. 


\begin{abstract}
The ant genus Octostruma Forel 1912 (Myrmicinae: Attini) is restricted to the Neotropics; their species occur from Mexico to Argentina, are predators of small arthropods and may nest in the soil, leaf litter and epiphytes. In the present work, the taxonomy of the genus is reviewed based in the examination of about 4.000 specimens. Recognize 43 Octostruma species, 34 species previously known are considered valid and redescribed and nine are recognized as new. Moreover, castes and sex of individuals not yet recorded (males, gynes and ergatoids) are described here for the first time for different species. New records expand the distribution known for most species and information about the biology of the species from label data and field observations, added to the little information in the literature are compiled in this work. A key to the worker of all species of the genus is provided.
\end{abstract}

Keywords: Ants, Myrmicinae, Octostruma, taxonomic revision, Neotropical region. 


\section{INTRODUÇÃO}

Para a resolução de qualquer questão biológica é fundamental a classificação das espécies em um sistema ordenado (Mayr et al., 1953). Ao longo de 280 anos de classificação taxonômica, foram catalogadas aproximadamente 1,2 milhões de espécies e, segundo uma estimativa realizada por Mora et al. (2011), 86\% das espécies terrestres e 91\% das espécies oceânicas ainda aguardam descrição.

Apesar de considerado um grupo relativamente bem estudado em relação aos outros insetos, as formigas não são exceção e novas espécies continuam sendo descobertas (Ward, 2007; Sosa-Calvo et al., 2013). Até o momento, Formicidae Latreille (1809) reúne 20 subfamílias (16 atuais e quatro extintas) e cerca de 15.000 espécies descritas (Bolton, 2014), sendo os insetos eussociais com maior riqueza de espécies e dominantes ecologicamente (Johnson et al., 2013). Estimativas sugerem que exista o dobro de espécies (Hölldobler \& Wilson, 1990; Ward, 2010). Nos últimos anos, espécies (e.g. Brandão et al., 2014; Fernandes et al., 2014) gêneros (e.g. Sosa-Calvo et al., 2013; Fernández et al., 2014) e uma subfamília (e.g. Rabeling \& Verhaagh, 2008) foram reconhecidos (Dlussky et al., 2004; Barden \& Grimaldi, 2013).

De todas as regiões biogeográficas do planeta, a região Neotropical é a que possui a maior diversidade e nível de endemismo de formigas (Fernández \& Sendoya 2004; Fisher 2010); grande parte do que ainda permanece desconhecido está concentrado nas florestas tropicais (Fernandéz \& Ospina, 2003) e acredita-se que cerca de $50 \%$ da mirmecofauna esteja associada à serapilheira nessas regiões (Delabie \& Fowler, 1995). O estudo da taxonomia de formigas de serapilheira tem sido crucial para o avanço da sistemática de formigas em geral (Longino, 2013).

A serapilheira é o hábitat de uma diversificada comunidade de pequenas formigas que, pelo seu tamanho relativamente pequeno e biologia ainda muitas vezes enigmática eram relativamente raras em coleções mirmecológicas até metade do século XX. Com o aperfeiçoamento das técnicas de coletas de formigas de serapilheira, os representantes de alguns gêneros (e.g. Basiceros Schulz 1906, Hylomyrma Forel 1912, Octostruma Forel 1912, Rogeria Emery, 1894 e Strumigenys Smith, 1860) passaram a ser coletados com frequência relativa muito maior. Aliado à isto, levantamentos de fauna de formigas estão sendo realizados pelo mundo inteiro, privilegiando ambientes até então pouco explorados, como por exemplo Caatingas (Brasil) (Neves et al. 2013; Ulysséa \& Brandão, 2013) e Florida Keys (Estados Unidos) (Moreau et al., 2014), o que tem contribuído para descrever 
comunidades de uma série de táxons até agora tido como raros, além de muitas espécies novas.

Neste ambiente de pequenas formigas comumente encontradas na serapilheira, está Octostruma Forel 1912, um gênero de formigas relativamente pequenas, predadoras de pequenos artrópodes, com a maior parte de suas espécies apresentando hábitos criptobióticos; que distribuem-se ao longo da região Neotropical, do México até a Argentina (Brown \& Kempf 1960, Wilson 1956, Wilson \& Brown, 1985).

As primeiras espécies conhecidas de Octostruma foram inicialmente descritas no gênero Rhopalothrix Mayr 1870. Em seu trabalho sobre as formigas da América do Sul, Mayr (1887) descreve duas espécies: $R$. petiolata, baseada em uma rainha e $R$. rugifer, baseada em uma operária, ambas coletadas em Santa Catarina, Brasil.

Em seguida, Emery (1888) descreve $R$. iheringi baseado em uma gine coletada no Brasil, e pela primeira vez menciona a presença de oito segmentos antenais.

Emery (1890) descreve $R$. simoni com base em operárias coletadas na Venezuela. Menciona a semelhança morfológica com o $R$. rugifer no que diz respeito ao formato da cabeça, das mandíbulas e a presença de oito segmentos antenais.

Emery (1894) em seu trabalho sobre a fauna Neotropical, descreve pela primeira vez a operária de $R$. petiolata com base em exemplares coletados em São Paulo (Brasil) e mais duas espécies, $R$. balzani com base em uma operária e uma gine coletada na Bolívia e $R$. batesi, com base em uma operária coletada no Amazonas (Brasil). Além disso, apresenta uma chave para as espécies de Rhopalothrix (incluindo as espécies com oito e nove segmentos antenais).

Forel (1899) descreve $R$. godmani com base em uma gine coletada no Panamá. Wheeler (1908) apresenta a lista de espécies coletadas por Alexande E. Weight em sua viagem à Jamaica e faz o primeiro registro de Rhopalothrix para a ilha descrevendo $R$. simoni var. wighti Wheeler. Wheeler atribui o status de variedade pelo tamanho relativamente maior em comparação aos exemplares descritos por Emery (1890).

O nome Octostruma aparece pela primeira vez na literatura no trabalho de Forel (1912) sobre as formigas mirmicíneas coletadas na região Neotropical. Forel propõe um novo subgênero para Rhopalothrix, reunindo as espécies com oito segmentos antenais e descreve ainda $R$. (O.) simoni r. spei, com base em operárias coletadas na Colômbia, atribuindo o status de raça por tratar-se de um indivíduo relativamente menor e a distingue de R. simoni var. weighti pelo tamanho relativamente menor, coloração escurecida e diferenças 
no formato da cabeça. Além disso, descreve $R$. (O.) truncata com base em uma gine coletada no Rio de Janeiro.

O subgênero Octostruma passa a incluir a seguinte configuração: (Rhopalothrix (Octostruma) balzani, R. (O.) batesi, R. (O.) iheringi, R. (O.) godmani, R. (O.) petiolata, $R$. (O.) rugifera, $R$. (O.) simoni, , $R$. (O.) simoni r. spei, $R$. (O.) simoni var. wighti e R. (O.) truncata).

Wheeler (1913) descreve $R$. (O.) lutzi com base em diversas operárias e uma gine coletadas na Dominica e a distingue de $R$. (O.) balzani pelo formato dos escapos antenais e das mandíbulas e de $R$. (O.) batesi pelas mesmas características e pelo tamanho relativamente menor.

Emery (1922) lista as espécies do subgênero Octostruma, sua distribuição e fornece uma diagnose, definindo o subgênero com mandíbulas com formato triangulares e antenas com oito segmentos.

No mesmo ano, Mann (1922), descreveu $R$. (O.) barberi e $R$. (O.) wheeleri, com base em operárias coletadas na Guatemala e comenta que $R$. (O.) barberi provavelmente pertenceria ao mesmo grupo com $R$. (O.) balzani e $R$. (O.) batesi e que $R$. (O.) wheeleri teria semelhanças com $R$. (O.) petiolata, introduzindo assim a primeira ideia de grupos para o então subgênero.

Weber (1934) em seu trabalho sobre as formigas Neotropicais descreve $R$. (O.) equilatera, com base em operárias coletadas na Nicarágua e inclui no mesmo grupo de $R$. (O.)balzani R. (O.) barberi e R. (O.) batesi.

Santschi (1936) descreve $R$. (O.) simoni spei var. sulcata, com base em uma operária do Panamá e a distingue de $R$. (O.) simoni por diferenças na coloração e na esculturação da superfície da cabeça.

O nome Octostruma como gênero é citado pela primeira vez na literatura no trabalho de Brown (1948), em uma lista de gêneros e subgêneros de Dacetonini, mas sem adicionar nenhum comentário sobre a mudança. Em seguida, o mesmo autor (1949) faz uma revisão de Dacetonini, excluindo alguns gêneros desta tribo, transferindo-os para a nova tribo, Basicerotini. Eleva formalmente Octostruma ao status de gênero, e utiliza como justificativa a presença dos oito segmentos antenais.

Brown \& Butting (1950) descrevem a venação de asas de vários gêneros de formigas para avaliar o potencial deste caráter no estabelecimento de uma filogenia de 
Formicidae; apresentam uma figura da asa anterior de uma gine de Octostruma, que posteriormente Brown \& Kempf (1960) afirmam pertencer à $O$. inca.

Brown \& Kempf (1960) apresentam a revisão mundial da tribo Basicerotini e a primeira revisão taxonômica para Octostruma, fornecendo uma diagnose para o gênero, redescrição das espécies conhecidas, sinonimizando $O$. godmani, $O$. simoni e suas variedades em $O$. iheringi e descrevendo três espécies (O. inca, $O$. stenognatha e $O$. rugiferoides.) Além disso, fazem a divisão em dois grupos de espécies (petiolata e balzani), propõem chave de identificação para todas as operárias das espécies e apresentam informações sobre a biologia das espécies com base nos dados de rótulo e a partir de inferências baseadas na morfologia das espécies.

Wheeler \& Wheeler (1977) descreveram a larva de $O$. inca, sugerindo uma diagnose para as larvas do gênero e Kluger (1978) estuda o aparelho de ferrão de O. balzani.

Wilson (1985) faz o registro de um fóssil de Octostruma, do âmbar da República Dominicana, que se encontra depositado na coleção do MCZC. Hölldobler \& Wilson (1986) apresentam um estudo detalhado dos pelos especiais de Basicerotini, concluindo que esses pelos auxiliam na camuflagem.

Perrault (1988) descreveu a espécie de $O$. betschi, com base em uma gine e uma operária coletadas na Guiana Francesa.

Baroni Urbani \& De Andrade (1994) consideram Basicerotini como sinônimo júnior de Dacetini. Em seguida, Bolton (1995) revive Basicerotini da sinonímia.

Em 1997, Palacio fez um levantamento das espécies de Octostruma da Colômbia e lista ao todo seis espécies, registrando novas ocorrências e descrevendo duas espécies (O. impressa e $O$. stenoscapa). Também forneceu informações sobre o hábito de forrageamento de $O$. impressa e uma chave para as espécies de Octostruma conhecidas da Colômbia.

Bolton (1998) comentou as apomorfias de Basicerotini, justificando sua aceitação como agrupamento monofilético. Ainda em 1998, Dietz apresentou sua dissertação de mestrado sobre a biologia e taxonomia de Octostruma, indicando a existência de oito espécies novas, esse estudo, porém, jamais foi publicado.

Em 2003, Bolton manteve sua proposta de Basicerotini como tribo, no que foi seguido por Dietz (2004) em sua tese de doutorado sobre uma revisão de Basicerotini.

Baroni Urbani \& De Andrade (2007), utilizando dados morfológicos, sinonimizam Basicerotini sob Dacetini, e todos os gêneros incluídos em Basicerotini sob 
Basiceros. Esta proposta não foi adotada por grande parte dos sistematas mirmecólogos, que alegaram necessidade de estudos mais aprofundados dos limites genéricos do grupo. Ainda neste trabalho, os autores descreveram uma espécie de Octostruma (O. onorei).

Makhan (2007), em um trabalho polêmico, descreveu Pyramica amrishi, que na verdade trata-se de uma Octostruma. Imediatamente, Bolton et al. (2008), com base nos poucos detalhes das imagens publicadas por Makhan (2007), transferiram a espécie de gênero e a sinonimizaram sob $O$. balzani com base no formato da cabeça e no padrão da pilosidade, tecendo diversas críticas severas ao trabalho.

Posteriormente, Longino (2013) apresentou uma revisão do gênero focando no material coletado na América Central, quando descreveu 19 espécies e elevou O. amrishi e O. lutzi à espécie.

Ward et al. (2015), utilizando dados moleculares, investigaram as relações evolutivas de Myrmicinae, reconhecendo seis das 25 tribos aceitas anteriormente e Basicerotini foi sinonimizada sob Attini.

Atualmente o grupo liderado pelo Dr. John Longino (University of Utah) está estudando as relações filogenéticas dos gêneros de Basicerotini (sensu Bolton, 2003) utilizando dados moleculares conforme discutido mais adiante neste trabalho.

Embora o gênero tenha sido alvo de uma revisão taxonômica recente ainda que parcial por Longino (2013) e os gêneros desta tribo estejam sendo estudado pelo grupo daquele especialista, o foco de seu trabalho foi a fauna da América Central. Assim, o material proveniente de coletas realizadas na América do Sul tem sido negligenciado e certas espécies carecem ser reestudadas. O fato da coleção de formigas do Museu de Zoologia da Universidade de São Paulo (MZSP) abrigar parte do material dos principais estudos do gênero (Kempf \& Brown, 1960; Dietz, 1998; 2004; Longino, 2013), integrando uma das coleções mais importantes para a região Neotropical (Brandão, 2000) contendo material de importantes coleções e de inúmeros projetos (concluídos e ainda em andamento) eu e meu orientador verificamos que existiam espécimes na coleção do MZSP provenientes de áreas jamais estudadas pelos autores de trabalhos anteriores, e que este acervo incluía táxons ainda não descritos, o que sugeria que o estudo das Octostruma sul-americanas poderia complementar o estudo de Longino (2013). 


\section{CONCLUSÃO}

Reconheço 43 espécies de Octostruma. Neste trabalho as 34 espécies previamente conhecidas são consideradas válidas e nove são reconhecidas como novas.

Os caracteres que melhor distinguem morfologicamente as operárias das espécies dentro do gênero são: dentição, formato da cabeça, quantidade e disposição dos pelos especializados, padrão de esculturação da superfície do dorso cefálico e do mesossoma e formato dos escapos antenais.

O conhecimento sobre gines e machos ainda permanece escasso e para maioria das espécies só é possível a identificação quando associadas às operárias.

A distribuição de Octostruma está restrita à região Neotropical, sendo que as espécies do gênero habitam ambientes mais conservados e contendo considerável acúmulo de serapilheira, as colônias são pequenas e formadas por algumas dezenas de operárias. Com base na literatura e na morfologia, a maior parte das espécies parece apresentar hábitos predadores generalistas. 


\section{REFERÊNCIAS}

Albuquerque, N.L. 2000. O gênero Oxyepoecus (Hymenoptera: Formicidae: Myrmicinae:

Solenopsidini). 121f. Dissertação de mestrado Instituto de Biociências da Universidade de São Paulo, São Paulo, Brasil.

Barden, P. \& Grimaldi, D. 2013. A new genus of highly specialized ants in Cretaceous Burmese amber (Hymenoptera: Formicidae). Zootaxa, (3681): 405-412.

Baroni Urbani, C. \& De Andrade, M. L. 1994. First description of fossil Dacetini ants with a critical analysis of the current classification of the tribe (Amber Collection Stuttgart: Hymenoptera, Formicidae. VI: Dacetini). Stuttgarter Beiträge zur Naturkunde. Serie B (Geologie und Paläontologie), (198): 1-65.

Baroni Urbani, C. \& De Andrade, M.L. 2007. The ant tribe Dacetini: limits and constituent genera, with descriptions of new species (Hymenoptera, Formicidae). Annali del Museo Civico di Storia Naturale "Giacomo Doria”, (99): 1-191.

Bolton, B. 1994. Identification guide to the ant genera of the world. Cambridge, Harvard University Press. 222 pp.

Bolton, B. 1995. A taxonomic and zoogeographical census of the extant ant taxa (Hymenoptera: Formicidae). Journal of Natural History, (29):1037-1056.

Bolton, B. 1998. Monophyly of the dacetonine tribe-group and its component tribes (Hymenoptera: Formicidae). Bulletin of the Natural History Museum. Entomology Series, (67):65-78.

Bolton, B. 2003. Synopsis and Classification of Formicidae. Mem. Am. Entomol. Inst. 370pp.

Bolton, B.; Sosa-Calvo, J.; Fernández, F. \& Lattke, J.E. 2008. New synonyms in neotropical myrmicine ants. Zootaxa, (1732): 61-64.

Brandão, C.R.F. 1991. Adendos ao catálogo abreviado das formigas da região Neotropical (Hymenoptera: Formicidae). Revista Brasileira de Entomologia, (35): 319-412.

Brandão, C.R.F. 2000. Major regional and type collections of ants (Formicidae) of the world and sources for the identification of ant species. In: Agosti, D.; Majer, J.D.; Alonso, L.E. \& 
Schultz, T.R. (Eds). Ants: Standard methods for measuring and monitoring biodiversity. Washington, Smithsonian Institution Press., p. 172-269.

Brandão, C.R.F.; Feitosa, R.M.; Powell, S. \& Del-Claro, K. 2014. Description of Cephalotes specularis n. sp. (Formicidae: Myrmicinae) - the mirror turtle ant. Zootaxa, (3796): 568-578.

Brandão, C.R.F.; Silva, R.R. \& Delabie, J.C. 2009. Formigas (Hymenoptera). In: Panizzi, A.R. \& Parra, J.R.R. (Eds). Bioecologia e nutrição de insetos. Base para o manejo integrado de pragas. Brasília, p. 321-369.

Brown, W.L. 1948. A preliminary generic revision of the higher Dacetini (Hymenoptera: Formicidae). Transactions of the American Entomological Society, (74):101-129.

Brown, W.L. 1949. Revision of the ant tribe Dacetini. I. Fauna of Japan, China and Taiwan. Mushi, (20): 1-25.

Brown, W. L. \& Kempf, W.W. 1960. A world revision of the ant tribe Basicerotini. Studia Entomologica, (3): 161-250.

Brown, W.L. \& Nutting, W.L. 1950. Wing venation and the phylogeny of the Formicidae. Transactions of the American Entomological Society, (75): 113-132.

De Andrade, M.L. \& Baroni Urbani, C. 1999. Diversity and adaptation in the ant genus Cephalotes, past and present. Stuttgarter Beitrage zur Naturkunde Serie B (Geologie und Palaontologie), (271): 1-889.

Delabie, J.H.C. \& Fowler, H.G. 1995. Soil and litter cryptic ant assemblages of Bahian cocoa plantations. Pedobiologia, ( 39): 423-433.

Dietz, B.H. 1999. Taxonomia e biologia de Octostruma Forel, 1912 (Hymenoptera: Formicidae: Myrmicinae: Basicerotini). 118f. Dissertação de mestrado - Instituto de Biociências da Universidade de São Paulo. São Paulo, Brasil.

Dietz, B.H. 2004. Uma revisão de Basicerotini Brown, 1948 (Formicidae: Myrmicinae), suas relações filogenéticas internas e com outras tribos dacetíneas (Dacetini e Phalacromyrmecini). 245f. Tese de Doutorado - Instituto de Biociências da Universidade de São Paulo. São Paulo, Brasil. 
Dlussky, G.M.; Brothers, D.J. \& Rasnitsyn A.P. 2004. The first Late Cretaceous ants (Hymenoptera: Formicidae) from southern Africa, with comments on the origin of the Myrmicinae. Insect Systematics \& Evolution, (35): 1-13.

Donisthorpe, H. 1943. A list of the type-species of the genera and subgenera of the Formicidae. Annals and Magazine of Natural History, (11)10: 617-688.

Emery, C. 1888. Formiche della provincia di Rio Grande do Sûl nel Brasile, raccolte dal dott. Hermann von Ihering. Bullettino della Società Entomologica Italiana, (19): 352-366.

Emery, C. 1890. Voyage de M. E. Simon au Venezuela (Décembre 1887 - Avril 1888). Formicides. Annales de la Société Entomologique de France, (6)10: 55-76.

Emery, C. 1894. Studi sulle formiche della fauna neotropica. VI-XVI. Bullettino della Società Entomologica Italiana, 26: 137-241.

Emery, C. 1922. Hymenoptera. Fam. Formicidae. Subfam. Myrmicinae. Genera Insectorum, (174): 95-206.

Emery, C. 1924. Hymenoptera. Fam. Formicidae. Subfam. Myrmicinae. Genera Insectorum, (174): 207-397.

Feitosa, R.S.M. 2007. Revisão taxonômica de Lachnomyrmex Wheeler, 1910 (Formicidae, Myrmicinae, Stenammini). 126f. Dissertação de mestrado - Faculdade de Filosofia, Ciências e Letras de Ribeirão Preto da Universidade de São Paulo, São Paulo, Brasil.

Feitosa, R.M. \& Brandão, C.R.F. 2008. A taxonomic revision of the Neotropical myrmicine ant genus Lachnomyrmex Wheeler (Hymenoptera: Formicidae). Zootaxa, (1890): 1-49.

Fernández, F. \& Ospina, M. 2003. Sinopsis de las hormigas de la región Neotropical. In: Fernandéz, F. (Ed.). Introducción a las hormigas de la región Neotropical. Bogotá, Instituto de Investigación de Recursos Biológicos Alexander Von Humbolt, p.49-64.

Fernandez, F.C. ; Feitosa, R.M. \& Lattke, J.E. 2014. Kempfidris, A New Genus of Myrmicinae Ants from the Neotropical Region (Hymenoptera, Formicidae). European Journal of Taxonomy, (85): 1-10.

Fernandez, F. \& Sendoya, S. 2004. List of Neotropical ants (Hymenoptera: Formicidae). Biota Colombiana, 5(3): 93. 
Fisher, B.L. 2010. Biogeography. In: Lach, L.; Parr, C.L. \& Abbott, K.L. Ant Ecology, Oxford University Press, Oxford. 402p.

Forel, A. 1899. Formicidae. Biologia Centrali-Americana Hym, (3): 1-169.

Forel, A. 1912. Formicides néotropiques. Part II. 3me sous-famille Myrmicinae Lep. (Attini, Dacetii, Cryptocerini). Mémoires de la Société Entomologique de Belgique, (19): 179-209.

Forel, A. 1917. Cadre synoptique actuel de la faune universelle des fourmis. Bulletin de la Société Vaudoise des Sciences Naturelles, (51): 229-253.

Harris, R.A. 1979. A glossary of surface sculpture. Occasional Papers of the Bureau of Entomology of the California Department of Agriculture, (28): 1-32.

Hölldobler, B. \& Wilson, E.O. 1986. Soil-binding pilosity and camouflage in ants of the tribes Basicerotini and Stegomyrmecini. Zoomorphology, (106): 12-20.

Hölldobler, B. \& Wilson, E.O. 1990. The Ants. Harvard University Press, Massachusetts. $732 \mathrm{p}$.

International Code of Zoological Nomenclature (ICZN). 1999. The International Trust for Zoological Nomenclature, London.

Johnson, B.R.; Borowiec, M.L.; Chiu, J.C.; Lee, E.K.; Atallah, J. \& Ward, P.S. 2013. Phylogenomics resolves evolutionary relationships among ants, bees, and wasps. Current Biology, (23): 2058-62.

Kempf, W.W. 1968. Miscellaneous studies on Neotropical ants. IV. (Hymenoptera, Formicidae). Studia Entomologica, (11): 369-415.

Kempf, W.W. 1972. Catálogo abreviado das formigas da região Neotropical. Studia Entomologica, (15): 3-344.

Kugler, C. 1978. A comparative study of the myrmicine sting apparatus (Hymenoptera, Formicidae). Studia Entomologica, (20): 413-548.

Longino, J.T. 2013. A revision of the ant genus Octostruma Forel 1912 (Hymenoptera, Formicidae). Zootaxa, (3699): 1-61. 
Longino, J.T. \& Boudinot, B.E. 2013. New species of Central American Rhopalothrix Mayr, 1870 (Hymenoptera, Formicidae). Zootaxa, (3616): 301-324.

Makhan, D. 2007. Six new Pyramica species from Suriname (Hymenoptera: Formicidae). Calodema, Supplementary Paper, (24): 1-7.

Mann, W.M. 1922. Ants from Honduras and Guatemala. Proceedings of the United States National Museum, (61): 1-54.

Mayr, E.; Linsley, E.G. \& Usinger, R.L. 1953. Methods and Principles of Systematica Zoology. McGraw-Hill, New York. 317 p.

Mayr, G. 1887. Südamerikanische Formiciden. Verhandlungen der Kaiserlich-Königlichen Zoologisch-Botanischen Gesellschaft in Wien, (37): 511-632.

Mora, C.; Tittensor, D.P.; Adl, S., Simpson, A.G.B. \& Worm, B. 2011. How Many Species Are There on Earth and in the Ocean? PLoS Biol., 9(8): 1-8.

Moreau, C.S.; Deyrup, M.A. \& Davis, L.R. 2014. Ants of the Florida Keys: species accounts, biogeography, and conservation (Hymenoptera: Formicidae). Journal of Insect Science, (14): 295 .

Neves, F.; Queiros-Dantas, K.S.; Rocha, W.D. \& Delabie, J.H.C. 2013. Ants of three adjacent habitats of a transition region between the Cerrado and Caatinga Biomes: The effects of heterogeneity and variation in canopy cover. Neotr Entomol, (42): 258-268.

Palacio, E. 1997. Hormigas de Colombia VI. Dos nuevas especies de Octostruma (Hymenoptera: Formicidae: Basicerotini). Caldasia, (19): 409-418.

Papavero, N. 1983. Fundamentos Práticos de Taxonomia Zoológica: coleções, bibliografia, nomenclatura. Museu Paraense Emílio Goeldi \& Sociedade Brasileira de Zoologia, Belém, 252 p.

Peeters, C. 2012. Convergent evolution of wingless reproductive across all subfamilies of ants, and sporadic loss of winged queens. Myrmecological News, (16): 75-91.

Perrault, G. H. 1988c. Octostrumma betschi, n. sp. de Guyane Française (Hymenoptera, Formicidae). Revue Française d'Entomologie, (10): 303-307. 
Rabeling, C.; Brown, J.M. \& Verhaagh, M. 2008. Newly discovered sister lineage sheds light on early ant evolution. Proceedings of the National Academy of Sciences, (105): 1491314917.

Santschi, F. 1936. Contribution à l'étude des fourmis de l'Amérique du Sud. Revista de Entomologia, (6): 196-218 .

Sosa-Calvo, J.; Schultz, T.R.; Brandão, C.R.F. ; Klingenberg, C.; Feitosa, R.M.; Rabeling, C.; Bacci, M.; Lopes, C.T. ; Vasconcelos, H.L. 2013. Cyatta abscondita: Taxonomy, Evolution, and Natural History of a New Fungus-Farming Ant Genus from Brazil. Plos One, (8), p.e80498.

Ulyssea, M.A. \& Brandão, C.R.F. 2013. Ant species (Hymenoptera, Formicidae) from the seasonally dry tropical forest of northeastern Brazil: a compilation from field surveys in Bahia and literature records. Revista Brasileira de Entomologia, (57): 217-224.

Ward, P.S. 2007. Phylogeny, classification, and species-level taxonomy of ants (Hymenoptera: Formicidae). Zootaxa, (1668): 549-563.

Ward, P.S.; Brady, S.G.; Fisher, B.L. \& Schultz, T.R. 2010. Phylogeny and biogeography of Dolichoderine ants: Effects of data partitionLing and relict taxa on historical inference. Systematic Entomology, (59): 342-362.

Ward, P.S.; Brady, S.G.; Fisher, B.L. \& Schultz, T.R. 2015. The evolution of myrmicine ants: phylogeny and biogeography of a hyperdiverse ant clade (Hymenoptera: Formicidae). Systematic Entomology, (40): 61-81.

Weber, N.A. 1934. Notes on neotropical ants, including the descriptions of new forms. Revista de Entomologia, (4): 22-59.

Wheeler, G.C. \& Wheeler, J. 1977. Supplementary studies on ant larvae: Myrmicinae. Transactions of the American Entomological Society, (103): 581-602.

Wheeler, W.M. 1908. The ants of Jamaica. Bulletin of the American Museum of Natural History, (24): 159-163.

Wheeler, W.M. 1913. Ants collected in the West Indies. Bulletin of the American Museum of Natural History, (32): 239-244. 
Wheeler, W.M. 1922. Ants of the American Museum Congo expedition. Bulletin of the American Museum of Natural History, (45): 1-11.

Wheeler, G.C. \& Wheeler, J. 1977. Supplementary studies on ant larvae: Myrmicinae. Transactions of the American Entomological Society, (103): 581-602.

Wilson, E.O. 1955. A monographic revision of the ant genus Lasius. Bull. Mus. Comp. Zool., (113): 1-201.

Wilson, E.O. 1956. Feeding behavior in the ant Rhopalothrix biroi. Psyche, (63): 21-23.

Wilson, E.O. 1985. Ants of the Dominican amber (Hymenoptera: Formicidae). Two new myrmicine genera and an aberrant Pheidole. Psyche, (92): 1-9.

Wilson, E.O. \& Brown, W.L. 1985. Behavior of the cryptobiotic predaceous ant Eurhopalothrix heliscata, n. sp. (Hymenoptera: Formicidae: Basicerotini). Insectes Sociaux, (31): 408-428.

Winston, J.E. 1999. Describing species. Practical taxonomic procedure for biologists. Columbia University Press, New York, 518p.

Yoshimura, M. \& Fisher, B.L. 2012. A revision of male ants of the Malagasy Amblyoponinae, with resurrections of the genera Stigmatomma and Xymmer. PloS ONE 7(3): $1-23$. 
